\title{
In memoriam: Jacques Leclerc
}

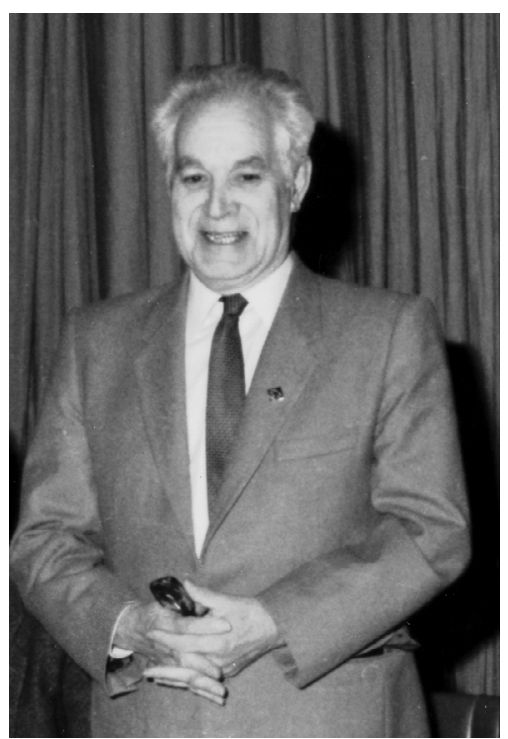

Jacques Leclerc est décédé le 17 décembre 2012 à l'âge de 91 ans.

Il avait le regard vif, de nombreux talents et de riches moyens d'expression.

Jacques Leclerc fut chargé de la Direction Métallurgique d'Usinor et il laisse de nombreux amis métallurgistes « orphelins » d'un modèle intellectuel et professionnel. Il fut un témoin et un acteur de décisions très importantes pour la Sidérurgie Française entre la fin 1945 et le début des années 80 .

Ingénieur de l'École Polytechnique (X42), il était le fils d'un ingénieur Centralien, chef d'Aciérie à Neuves-Maisons, puis à Montluçon (Compagnie des Forges de ChâtillonCommentry et Neuves-Maisons). Comme d'autres ingénieurs de sa génération, il n'avait pas pu intégrer son école en 1942 et avait du attendre l'année 1944 pour poursuivre ses études. Il avait mis à profit cette période d'attente pour travailler à Montluçon où son père était alors en fonction. Son père pour lequel il avait beaucoup de considération l'avait ainsi amené à découvrir la Sidérurgie et la recherche (il gardait encore les carnets de son père sur lesquels étaient reportées entre-autres des coupes de divers lingots d'acier). Il était rentré à Denain-Anzin, devenu ensuite Usinor, sous la présidence de René Damien, dont il disait qu'il avait reçu la mission de servir en devenant «son métallurgiste» [1]. Au cours de sa carrière qui l'a conduit à exercer cette mission au plus haut niveau, il n'a cessé d'approfondir et de développer avec une grande passion les fonctions et les différents volets de cette mission : études et recherches dans le domaine des procédés et des produits, définition des gammes de fabrication et du contrôle de la qualité, méthodes d'investigation, liaisons avec la clientèle... Il a réussi à donner à ces métiers un rayonnement de premier plan et à les rendre attractifs pour tous ceux qui ont eu la chance de travailler avec lui. L'exercice de ce métier qu'il a valorisé et rendu « incontournable » l'a conduit à participer à de nombreuses décisions. 


\section{Une vision globale et intégrée sur l'élaboration et la qualité}

Jacques Leclerc couvrait un domaine très vaste et détaillé sur toute la filière de production depuis la fonte, jusqu'au produit chez le client (le tube, la tôle épaisse sur plateforme pétrolière, la tôle mince emboutie pour l'automobile et pour d'autres applications, etc.). Il a contribué à l'évolution des procédés et à l'assurance d'un bon service client par la prise en compte de l'évolution des spécifications et des besoins des utilisateurs, en tenant compte des limites de possibilité des outils de production.

Il a conduit ses équipes placées sous la Direction Métallurgique à l'optimisation de la composition chimique (spécification des teneurs en $\mathrm{S}$ et $\mathrm{P}$ et des additions de $\mathrm{Mn}, \mathrm{Nb}, \mathrm{V}$, Mo, Ce, B... ) et des conditions de laminage (température de défournement, température de fin de laminage, température de bobinage, etc.) de façon à obtenir au meilleur coût de production les meilleures caractéristiques mécaniques, en tenant compte de la qualité globale des produits (santé interne, aspect de surface, soudabilité, aptitude à l'emboutissage, aptitudes diverses à l'emploi).

La prise de commande de tôles à tubes de grade élevé pour le transport du gaz et de plaques en acier normalisé pour la construction des plateformes pour le champ de Frigg dans le courant des années 70 illustre bien cette démarche [2]. Les aciers à basse teneur en $S$ et $P$ étaient traités au Cérium pour assurer la formation d'inclusions " globulaires » de sulfure de Mn. Avec l'appui des divers services concernés, les services de recherche et développement, aussi bien que les services de production, les équipes ont réussi à optimiser le traitement de désulfuration sur la fonte et sur l'acier, et à trouver le meilleur mode de traitement des inclusions par injection de fil fourré au Cérium dans le moule et le répartiteur de la coulée continue [3], et le traitement a été effectué par la suite par injection de SilicoCalcium en poche [4]. L'élaboration et le traitement aval (conditions de refroidissement) devaient être conduits de façon à contrôler la teneur en hydrogène par l'optimisation des paramètres d'élaboration (addition des ferrailles notamment), et par refroidissement lent des brames et des tôles. Par la suite ce processus sera optimisé encore par le traitement sous vide. Les études des propriétés s'étendaient de la tôle jusqu'au tube en intégrant le procédé aval de fabrication [5] et des mécanismes complexes d'évolution des propriétés. Les études de soudabilité ont démontré la faisabilité de tubes répondant aux normes API X70 dès cette première commande pour le champ de Frigg [6].

Il intégrait les connaissances scientifiques et techniques détaillées qui étaient nécessaires dans ce processus d'amélioration continue et d'innovation. Il entretenait des contacts réguliers avec les laboratoires universitaires (Ecoles des Mines, Facultés) ou les centres de recherche associés (Irsid, Cetim, CRM, Ctif, etc.). Le laminage contrôlé des tôles à tubes que nous présenterons plus loin est un bon exemple de la coopération entretenue avec les équipes de 1'Irsid à Saint-Germain-en-Laye et 1'Irsid en a donné un témoignage [7]. Il était un défenseur de la culture scientifique et il n’hésitait pas à coopérer de façon ouverte avec ses collègues, ses fournisseurs et ses clients pour la communication des résultats de la recherche et l'optimisation des fabrications.

\section{La Coulée Continue : " Un grand saut sans petit pas » [8]}

Les premiers développements de la Coulée Continue ont été effectués à l'échelle pilote et semi-industrielle entre 1940 et 1960 .Un historique précis du développement de la Coulée Continue a été écrit par Manfred Wolf dans la Revue de Métallurgie [9-11]. L'Allemagne (Mannesman, Forges et Aciéries de Dillingen), l'Autriche (Böhler), la France (CAFL, FivesLille, Forges d'Allevard, SAFE) furent les pionniers en Europe. En 1965, il n'y avait en France que 4 installations de coulée continue de billettes et de blooms pour le laminage de produits longs. Une installation pilote de coulée continue centrifuge de ronds était également construite à Imphy. Les premières difficultés ayant été résolues, le développement de la 
coulée continue rencontrait quelques grandes difficultés, en particulier pour la production de produits plats destinés à l'industrie automobile.

Ces fabrications étaient réalisées auparavant par la coulée dans des lingotières classiques d'acier "effervescent » ou «stabilisé » avec un savoir-faire approprié pour le contrôle de l'oxydation du métal. Une grande qualité de peau était attribuée aux brames obtenues, ce qui permettait en particulier de réaliser des aciers pour l'automobile aptes à l'emboutissage et en aspect $Z$ (une qualité de surface appropriée à la carrosserie visible). Il est apparu rapidement que ces aciers ne pouvaient pas être coulés en continu. Jacques Leclerc et ses équipes avaient fait l'expérience à Denain du laminage de brames russes en acier effervescent élaborées à Donetsk et à Novolipetsk. Des visites en Russie ont confirmé que le procédé n'était pas bien maîtrisé et la qualité des produits obtenus après laminage à Denain n'était pas satisfaisante.

Malgré l'échec de l'essai de laminage des brames en acier effervescent, Jacques Leclerc et les équipes d'Usinor ont continué à instruire le dossier de la coulée continue. Une première machine a été installée dans l'Aciérie 1 de l'usine de Dunkerque - décidée en janvier 1968 et démarrée le 20 janvier 1971 [12] - pour la production de brames destinées à la tôlerie forte. À partir des premiers essais sur la filière tôles minces et des nombreuses visites effectuées aux USA et en Allemagne, il est apparu la possibilité de réaliser des aciers pour la carrosserie automobile visible (en aspect Z) à partir d'aciers calmés à l'Aluminium. Il fallait alors compléter l'investissement de machines de coulée continue par celui d'une installation de «scarfing » permettant d'éliminer de la première peau des inclusions éventuelles d'oxydes cette précaution a pu être supprimée par la suite.

Jacques Leclerc a eu alors le mérite d'orienter le choix en faveur de la coulée continue pour l'Aciérie No. 2 de l'usine de Dunkerque, 1ère aciérie au monde avec l'Aciérie de Nippon Steel à Oita à couler son acier à $100 \%$ en coulée continue. Il s'agissait de doubler la capacité de l'usine (de 4 à $8 \mathrm{Mt}$ ) sans investir un nouveau slabbing (et avec une économie d'investissement, un gain de mise au mille et de coût par suppression d'une étape dans le laminage). Il disait [1] que «la décision avait été prise dans l'avion le 15 mai 1969, de retour d'un voyage aux USA, avec le Directeur Général d'Usinor, le Directeur de l'usine et le Chef d'Aciérie de l'usine de Dunkerque, le jour même où l'on faisait un premier pas sur la lune » (c'est son propre texte que l'on a emprunté - en réalité, le premier pas sur la lune a eu lieu le 20 juillet mais c'était une façon semi-humoristique sans doute de présenter cette décision importante, en négligeant un peu la précision sur les dates). Les machines de coulée continue de l'Aciérie No. 2 ont démarré le 26 octobre 1972, peu de temps après le démarrage des convertisseurs [13]. Comme Jacques Leclerc le disait, c'était une "grande aventure » qui démarrait et un «saut » auquel il fallait croire. Il rendait hommage à ceux qui l'avaient aidé à faire ce saut, en particulier à Von Emde chez Mannesmann et à tous ses collègues français chez Usinor.

Assez curieusement, M. Wolf a négligé ou oublié de mentionner dans son historique ce grand pas effectué par Usinor-Dunkerque pour l'utilisation de la Coulée Continue. Jacques Leclerc tenait à corriger cet oubli et par le présent document nous pourrions également y contribuer.

Par la suite, Jacques Leclerc a contribué avec toute l'équipe des ingénieurs de Dunkerque au développement et au perfectionnement de la Coulée Continue [14]. De nombreux progrès ont été effectués sur la $1^{\text {ère }}$ machine de l'Aciérie No. 1, en particulier dans le domaine des réfractaires, avec le développement de la quenouille monobloc et de la busette immergée en alumine graphitée [15], dont la première mise au point (avec un réfractaire en Glassrock) revenait à M. Gosselin de la SAFE (Société des Aciers Fins de l'Est à Hagondange). Il a demandé à l'Irsid d'étudier les poudres de couverture : il fallait trouver des poudres dont les propriétés physico-chimiques qui étaient connues pour assurer la qualité des produits, devaient rester stables dans le temps. Les développements ont conduit la société Anzinminéraux à fournir des poudres de couverture réalisées avec des approvisionnements en matières premières connus et constants dans le temps, adaptées à différentes conditions de coulée $[16,17]$. 
Avec l'Irsid, qui avait effectué une première mise au point du brassage électromagnétique dans le refroidissement secondaire des billettes et des blooms à la SAFE pour améliorer la ségrégation axiale, il a cherché une solution pour améliorer également la surface et la qualité de peau. Cela a conduit à la mise au point du brassage en lingotière et à la création de Rotelec, société chargée du développement de toutes les techniques de brassage. La technique était d'abord appliquée aux produits longs et de nombreuses recherches ont été réalisées ensuite pour étendre les applications aux produits plats, en particulier à l'usine de Dunkerque [18]. Jacques Leclerc a été le président de Rotelec au début des années 1980.

\section{Le Vide : "Pourquoi faire? "}

Comme il l'avait eu pour la Coulée Continue, Jacques Leclerc avait l'intuition qu'une grande usine comme Dunkerque devait avoir une installation de traitement sous vide et il avait envoyé plusieurs ingénieurs en visite pour voir ce que l'on faisait ou pourrait faire avec une telle installation. A Isbergues, chez Thyssen et chez Hoesch dans la Ruhr, le vide servait à traiter des aciers au Silicium bas Carbone pour tôles électriques ou à déshydrogéner des aciers pour tôles fortes. En 1981, un accord avait permis de faire des visites dans toutes les usines du groupe NSC et laissait entrevoir la possibilité de faire des aciers ULC (Ultra Low Carbon) dont la pureté pouvait être exploitée métallurgiquement. Ces premières visites ont conduit les ingénieurs à justifier le vide pour l'élaboration des aciers au Silicium et le dégazage des tôles fortes d'une part et aussi à l'installer dans l'Aciérie No. 2 pour y traiter des aciers à très bas carbone pour la filière tôles minces. L'intérêt des aciers IFS n'était pas encore connu au stade industriel.

La première coulée du RHOB dans l'Aciérie No. 2 de l'usine de Dunkerque a été réalisée en Novembre 1984 [19]. Les premiers essais de coulée d'acier IFS (Interstitial Free Steel) en 1985 ont montré qu'il y avait un gros intérêt à élaborer ces aciers pour la galvanisation. Ces aciers pouvaient en effet se passer du traitement de survieillissement que l'on devait pratiquer après la galvanisation. Ils conduisaient ainsi à une meilleure qualité avec un coût plus raisonnable que par la filière précédente. Le vide à Dunkerque avait trouvé sa justification industrielle. Et il apportait en même temps un élan décisif au développement des aciers galvanisés pour l'industrie automobile.

\section{Les aciers galvanisés : fallait-il du zinc sur une face ou sur deux faces?}

Dès 1975, début de la demande d'aciers revêtus résistants à la corrosion par les constructeurs automobiles en particulier aux Etats Unis, Jacques Leclerc soutient des recherches dans l'objectif de trouver des solutions à partir d'acier galvanisé.

Avec le développement du Monogal ${ }^{\circledR}$ (Acier galvanisé avec une face revêtue de zinc métallique et l'autre face brossée revêtue uniquement d'une très fine couche d'alliage Fe$\mathrm{Zn}$ ) aux Laminoirs de Strasbourg, il va participer activement à la vente du brevet à d'autres sidérurgistes et à faire produire le Monogal à Strasbourg et sur les installations de Galvanor à Montataire dont il devient le président.

$\mathrm{Au}$ fil des années, les constructeurs qui voulaient d'abord utiliser du zinc uniquement en face non visible, prennent conscience que cela ne suffit pas à répondre à la demande de résistance à la corrosion, et s'orientent vers l'emploi de tôles revêtues sur les 2 faces. L'expérience acquise lors de la production du Monogal ${ }^{\circledR}$ va conduire sous l'impulsion de Jacques Leclerc au développement de $l^{\prime} E x t r a g a l{ }^{\circledR}$, acier galvanisé 2 faces pour l'automobile qui reste encore aujourd'hui la principale solution utilisée par les constructeurs automobiles [20].

\section{Le laminage contrôlé : un processus clé pour l'optimisation de la filière}

Avant 1960 le laminage contrôlé concernait essentiellement les tôles à tubes à l'exception de tôles navales faites par KN Hoogovens à Ijmuiden du fait que cette usine n'avait pas de four pour le recuit de normalisation. 
Les nuances contenant du niobium étaient livrées à l'état brut de laminage après une forte réduction à basse température d'où le terme de «laminage contrôlé ».

La croissance du marché des gazoducs aux Etats Unis mais aussi aux Pays-Bas puis en mer du Nord a conduit à des évolutions sur les diamètres, donc les largeurs de tôles et sur les propriétés passant des spécification de la norme API X52 à X60 et X65 avec de nouvelles exigences en ténacité et soudabilité.

Suite à la fusion Usinor Lorraine Escaut ce marché est devenu stratégique pour le nouvel Usinor. Jacques Leclerc était bien au courant d'un ensemble d'études métallurgiques de l'IRSID sur d'une part la recristallisation à chaud en cours de laminage [21], le durcissement par le niobium et d'autre part la soudabilité et la ténacité. Les études métallurgiques et les études sur l'automatisation des laminoirs Quarto [22] étaient coordonnées et intégrées dans un même ensemble qui a débouché sur des applications industrielles et des brevets (modèle Plate) [23].

Il a non seulement demandé d'accélérer ces études mais il a aussi assuré une coordination poussée avec les usines en provoquant de nombreuses réunions afin de convaincre les exploitants de la faisabilité du laminage contrôlé sur des laminoirs (Quartos de Longwy et Dunkerque) qui n'étaient pas à priori conçus pour travailler à basse température avec des efforts importants.

Ayant tout de suite compris le point capital de l'additivité des passes en cas de non recristallisation en présence de niobium Jacques Leclerc a parfaitement orchestré les efforts pédagogiques des chercheurs vis-à-vis de l'ensemble des lamineurs jusqu'aux grands patrons.

Il a fallu assurer la crédibilité des schémas de laminage avec des temps d'arrêts parfois longs et l'optimisation des enchainements de brames. Ainsi quand est arrivée la commande de tubes sous-marins pour le champ de Frigg en tôle épaisse, Usinor était prêt avant la concurrence. Vu le tonnage très important à livrer dans un délai réduit, Jacques Leclerc a par précaution - négocié avec les Forges et Aciéries de Dillingen la prise en charge d'une partie de la commande, et il a ouvert une coopération technique avec F.A. Dillingen dans le domaine des tôles à tubes [24,25].

Il s'est lui-même beaucoup impliqué dans la promotion du laminage contrôlé de ce type en suggérant des publications en Anglais en particulier pour la conférence Microalloying 75 avec 3 présentations en Anglais : une d'Usinor sur la mise au point des nuances, une de l'IRSID plus théorique et une (commune) sur la soudabilité [2].

Par la suite le développement du laminage contrôlé pour de nouvelles nuances et surtout d'autres applications (ponts, réservoirs de stockage, chaudronnerie) a nécessité encore de nombreux efforts d'explications et d'essais pour convaincre les clients, les organismes de contrôle et de certification et les administrations.

Pour faire connaitre ces avancées, Jacques Leclerc a fait déposer une marque Multiphi qui désignait des schémas de laminage plus sophistiqués avec 2 arrêts en cours de laminage si nécessaire. Cette démarche entérinait en fait pour la première fois le contrôle de toutes les passes et le passage du laminage contrôlé aux traitements thermomécaniques proprement dits.

Les grades devenant de plus en plus élevés (X70 et plus) avec des épaisseurs plus fortes il fallait accélérer le refroidissement après la fin du laminage et cela avait été bien anticipé par Jacques Leclerc mais malheureusement la technique d'arrosage n'était pas prête en France et on s'est à ce moment là orienté vers la trempe directe avec le RAC; cette technique fut intéressante mais exigeant un revenu elle fut moins prometteuse que le refroidissement accéléré mis au point peu après au Japon.

L'essor des traitements thermomécaniques est un bon exemple de la conjonction de beaucoup de connaissances métallurgiques sur les microstructures, les propriétés et les technologies de l'aciérie aux laminoirs pour lesquelles Jacques Leclerc a joué le rôle de chef d'orchestre. 


\section{Un rôle humain de grande importance}

Narrateur infatigable, passionné par l'histoire des procédés et de la vie des usines, il savait rendre attractif ce qui pouvait paraître fastidieux ou complexe. Il faisait appel à toutes ses connaissances métallurgiques pour faire une synthèse et s'approprier ce qui était présenté par les différents courants qui s'exprimaient. C'était un « animateur suivi » aussi bien sur le tas en usine que dans les laboratoires et il jouait ce rôle d'animateur dans les deux domaines industriel et scientifique.

Georges Delbart, premier Directeur de l'Irsid, lui avait déclaré qu'il serait plus utile à la recherche et à la vie de l'Irsid en restant Usinor plutôt qu'en devenant un responsable du centre de recherche qu'il aurait aimé diriger lui-même. S'étant approprié cette mission que lui avait donnée Delbart, il a fait confiance à l'Irsid et a orienté ses recherches sans chercher à développer des moyens indépendants pour sa société. Les Directeurs Généraux successifs de l'Irsid, Marc Allard et Lucien Coche ont apprécié son aide et ses contributions. Pendant de nombreuses années, il a su coopérer avec ses collègues des sociétés Sollac et Sacilor pour donner des orientations au Comité d'Organisation Technique de l'Irsid [7].

Il a joué ce rôle d'animateur également au sein de la Société Française de Métallurgie (SF2M maintenant), en 1973 et 1974, à la suite de Charles Crussard. Il considérait que les contacts que la SF2M assurait entre les métallurgistes de sociétés «clients » et de sociétés « fournisseurs » $d^{\prime}$ une part, entre « industriels » et « universitaires » d'autre part étaient le point fort de cette société savante dont le développement était indispensable [26].

Pour l'Irsid et pour Usinor, il a joué un rôle important dans la gestion des cadres techniques, en éclairant les choix et en décelant les véritables vocations de chacun. Il a exercé ce talent non seulement dans le cadre de la Direction Métallurgique, mais aussi pour les Directions Générales qui étaient à son écoute. Il avait le don de parler, d'écouter et de découvrir les motivations et les orientations les plus prometteuses pour chacun des ingénieurs qu'il était amené à rencontrer, en particulier les plus jeunes. Pour engager la conversation, il faisait parfois appel à son équation favorite qui rapprochait connaissances, besoins et progrès [27]. Après cette ouverture, ses collaborateurs étaient entraînés et pouvaient donner libre cours " aux espoirs et illusions, aux efforts et engagements qui pouvaient donner un sens à la vie », selon ses propres expressions En puisant dans la "pépinière » de l'Irsid et en valorisant les jeunes recrues de sa Direction Métallurgique, il a contribué à mettre à la bonne place un bon nombre d'experts et managers nécessaires à l'encadrement technique d'Usinor. Ils ont fait la preuve du bon choix qui avait été fait.

Jacques Leclerc était resté attaché à la région Nord où il avait commencé sa carrière dans l'usine sidérurgique intégrée de Denain. Il avait conservé les bureaux de la Direction Métallurgique dans l'usine proche de Valenciennes jusqu'à sa retraite. Il était souvent sur les routes pour aller là où l'intérêt et l'utilité de sa présence le demandaient. Il changeait souvent de programme et arrivait tard le soir pour faire un compte-rendu à son épouse qui l'attendait. En dehors de sa vie professionnelle qui a été très riche, Jacques Leclerc aimait s'occuper de ses nombreux amis et de sa famille. Il a fondé le club Rotary à Denain, et avec ce club a soutenu des actions en faveur des handicapés mentaux pour l'association des «Papillons Blancs». Il affectionnait particulièrement ses cinq enfants, dont il a suivi la scolarité, ses douze petits enfants et ses cinq arrièrepetits enfants auxquels il a consacré du temps et beaucoup d'attention. Il hébergeait souvent l'un d'eux dans la pièce qui lui servait de bureau à Paris.

Jacques Leclerc avait reçu la médaille Portevin de la SF2M (en 1979) et il était Chevalier de l'Ordre National du Mérite.

$C^{\prime}$ est un grand ami et un exemple qui nous a quittés.

Robert Alberny, Michel Jeanneau et Marc Grumbach

Avec les contributions de Gilles Garrigues, Michel Lahousse, Alain Coolen, Suzanne Mathieu, etc. 


\section{Bibliographie}

cette bibliographie contient des articles publics et privés signés par Jacques Leclerc ainsi que des articles rédigés et signés par d'autres ingénieurs dans le cadre d'actions qu'il avait particulièrement engagées ou suivies.

\section{Références}

[1] J. Leclerc, Revue de Métallurgie 99 (2002) 599-613

[2] M. Lafrance, Caron, Lamant, J. Leclerc, Use of microalloyed steels in the manufacture of controlled plates for pipes, Symposium Microalloying 75, New York, pp. 14-20

[3] F. Sauvage, D. Senaneuch, R. Douchy, Revue de Métallurgie - CIT 82 (1985) 31

[4] M. Jeanneau, M. Poupon, D. Senaneuch, M. Devaux, J.-P. Motte, Revue de Métallurgie - CIT 78 (1981) 525-529

[5] A. Coolen, M. Grumbach, M. Poupon, L. Roesch,Revue de Métallurgie - CIT 80 (1983) 261

[6] J. Liegeois, Revue de Métallurgie - CIT 76 (1979) 569

[7] Irsid, Communication Irsid

[8] J. Leclerc, La Coulée Continue 1964/1970, une aventure vécue, un grand saut sans petit pas Communication privée

[9] M. Wolf, Revue de Métallurgie - CIT 91 (1994) 75-98

[10] M. Wolf, Revue de Métallurgie - CIT 91 (1994) 445-588

[11] M. Wolf, Revue de Métallurgie - CIT 91 (1994) 589-608

[12] J.-P. Boyer, Revue de Métallurgie - CIT 68 (1971) 709-723

[13] J.-P. Boyer, C. Meens, M. Cagnet, J. Déchaux, C. Villard, Revue de Métallurgie - CIT 70 (1973) 801-822

[14] J. Leclerc, R. Martin, D. Amory, Revue de Métallurgie - CIT 70 (1973) 97

[15] D. Senaneuch, M. Poupon, Revue de Métallurgie - CIT 78 (1981) 525-529

[16] P.V. Riboud, Y. Roux, L.D. Lucas, et al., Fachberichte Huttenpraxis Metallweiterverarbeitung 19 (1981) 859-869

[17] P.V. Riboud, M. Larrecq, Revue de Métallurgie - CIT 78 (1981) 29

[18] R. Pouchelle, Revue de Métallurgie - CIT 85 (1988) 255-262

[19] M. Lahousse, L. Cussac, Revue de Métallurgie - CIT 84 (1987) 535-543

[20] M. Chilaud, B. François, R. Wang, B. Dubreuil, J.J. de Villeroche, Revue de Métallurgie - CIT 83 (1986) 599-606

[21] A. Le Bon, Revue de Métallurgie - CIT 76 (1979) 149

[22] G. Fritsch, G. Haralamb, J.-C. Illaire, J.-P. Schiavon, J. Bouvard, Revue de Métallurgie - CIT 70 (1973) 213-223

[23] B. Fazan, Revue de Métallurgie - CIT 76 (1979) 253

[24] Irsid, Usinor, Forges de Dilling, Revue de Métallurgie - CIT 72 (1975) 297-328

[25] A. Coolen, M. Jeanneau, Revue de Métallurgie - CIT 77 (1980) 711-724

[26] J. Leclerc, Interfaces, Transformations en continu : reflets et perspectives-, Discours du 29 janvier 1975 à la SF2M

[27] J. Leclerc, $B+C=P+Q$, Besoins, Connaissances et Progrès, Communications Privées - lettres à $B$. Rogy et M. Lafrance/G. Garrigues (1994) 